\title{
Characteristics and Manufacture of Spherical Smokeless Powders
}

\author{
Fernanda Diniz Botelho, Erick Braga Ferrão Galante , Álvaro José Boareto Mendes
}

\begin{abstract}
Smokeless propellants have been studied and manufactured for many decades. They can exist in various physical forms and also can have different properties according to the use of each propellant. One important form of smokeless powders is the ball powder, which has spherical grains. The manufacture process of the ball powder has many advantages over the usual way to manufacture a smokeless powder. For example, unstable and even deteriorated nitrocellulose, after being stabilized again, can be used to produce spherical powders. Also, the process to produce them requires less water and energy, which can result in a cheaper way to manufacture powders. In this review, the spherical powders manufacturing process is described, as well as the characteristics and advantages of this specific form of a double-base powder.
\end{abstract}

KEYWORDS: Double-base propellants, Single-base powders, Spherical powders, Smokeless powders.

\section{INTRODUCTION}

Smokeless powders are a class of propellants that were developed in the late $19^{\text {th }}$ century to replace black powder. There are patents regarding smokeless powder dating back to the beginning of the $20^{\text {th }}$ century (Piper 1953; Boddicker 1940). The term "smokeless" refers to the minimal residue left in the gun barrel following the use of smokeless powder (Heramb and McCord 2002).

Smokeless propellants can be found in many physical forms: single or multi-hole cylinders, thin sheets, strips and others. An important type of smokeless propellants that has been studied and manufactured for more than 70 years is the spherical powder, also called ball powder. This variation of smokeless propellants was first studied by Fredrich Olsen and peers (Olsen et al. 1936, 1939; Olsen and Tibbitts 1938; Olsen 1942). By the early 1930s, the first patent about the manufacture spherical powder was published (Valença et al. 2013). It was noticed that spherical powders had some advantageous characteristics such as progressive burning rate and high loading density (U. S. Army 1964), which made it possible to use them in small ammunition guns.

The history of explosives started with black powder, which was probably the first explosive to be manufactured. Accidents involving black powder were reported in $220 \mathrm{BC}$ in a Chinese document (Akhavan 2004; Valença et al. 2013), when some alchemists mixed potassium nitrate, sulfur as well as charcoal, and a big explosion was the result. Black powder was used for blasting for many years, but the limitations of this explosive became apparent in the $19^{\text {th }}$ century. For example, mining and tunneling operations using black powder were very difficult (Akhavan 2004).

\footnotetext{
1. Instituto Militar de Engenharia - SE/5 - Seção de Engenharia Química - Rio de Janeiro/RJ - Brazil

Author for correspondence: Erick Braga Ferrão Galante | Instituto Militar de Engenharia - Seção de Engenharia Química | Praça General Tibúrcio, 80 | CEP. 22. 290-270 - Urca - Rio de Janeiro/RJ - Brazil | Email: erickbfgalante@gmail.com

Received: 02/15/2015 | Accepted: 11/02/2015
} 
A second big event in the history of explosives was the discovery of sulphuric and nitric acids. With these two substances, it was possible to nitrate many compounds, making new ones, which were frequently very unstable and would explode, so they could eventually replace black powder. In 1861, Alfred Nobel built a small manufacturing plant to make nitroglycerine, a liquid with an explosive nature. Nitrocellulose was discovered a few years after nitroglycerin (Valença et al. 2013), and then scientists around the world developed many other important explosive substances such as trinitrotoluene and ammonium nitrate.

The great number of new explosives resulted in the replacement of black powder as a blasting explosive. However, this compound is still used in fireworks and in rockets as fuel (Valença et al. 2013).

After the development and manufacture of many explosive compounds, it became necessary to separate them into groups in accordance with their properties, for example, their burning characteristics and stability. A classification by performance and uses of explosives divides those substances into three groups: primary explosives, secondary explosives and propellants. Primary explosives differ from secondary explosives in that they undergo a very rapid transition from burning to detonation and can transmit the detonation to less sensitive explosives (Cooper 1996; Akhavan 2004). Secondary explosives are less sensitive than primary explosives and do not initiate by heat or shock. Propellants are combustible materials that do not usually detonate; they only burn in high velocity (which is defined as deflagration). The most common way of initiate them is by using a blasting cap generating heat (Valença et al. 2013; Akhavan 2004; Cooper 1996). Propellants may be named as powders or gunpowder by some literature. Regarding United Nations risk classifications, propellants are hazardous goods classified in general as 1.3C, but depending upon conditions, they can burn to detonation and are classified as 1.1C (United Nations 2015).

Nitrocellulose and nitroglycerin are energetic bases for smokeless powders, because they can be used as propellants, and their burning produces much less residues than that of black powder (Valença et al. 2013; Heramb and McCord 2002). All smokeless powders can be placed into one of three different classes according to the chemical composition of their energetic ingredients. A single-base powder contains nitrocellulose, whereas a double-base powder contains nitrocellulose and nitroglycerine. Nitroguanidine can also be included as a third energetic base, forming a triple-base propellant (Valença et al. 2013; Akhavan 2004; Heramb and McCord 2002; Cooper 1996). In some cases, nitroglycerine can be replaced by nitroglycol because it is more stable at lower temperatures than nitroglycerine (Valença et al. 2013).

\section{SPHERICAL SMOKELESS POWDERS CHARACTERISTICS}

Spherical smokeless powders were first studied by Fredrich Olsen and peers (Olsen et al. 1936, 1939; Olsen and Tibbitts 1938; Olsen 1942). Olsen worked for Western Cartridge Company, an ammunition industry (Hodgdon Powder Co. 2014), and he was studying new ways to stabilize nitrocellulose because its decomposition was a problem during World War I. Olsen observed that a mixture of nitrocellulose, ethyl acetate and water, when agitated, tended to form small spheres that did not last when the agitation was interrupted. This fact motivated Olsen to start studying new ways to manufacture a propellant in a spherical shape.

The spherical powder manufacture process provided many advantages over common extruded-type powders that were quickly taken advantage of by ammunition factories. Its key advantages include: stable long life; high density (ideal for rifle calibers); adjustable chemistry, which allows the powder to be tailored to specific cartridge applications through chemistry and geometry; and lower flame temperature, which allows longer barrel life in service (Barzanjy 2011; Tao et al. 2010).

The spherical powder is not only substantially spherical in shape but uniformly dense. It, therefore, has advantages not only from a ballistic standpoint but also from a loading standpoint. Spherical grains of the same size and composition burn at the same rates. That is impossible with angular or oblong grains due to their varying shapes having different volumes, even though all may pass through the same screen opening (Olsen et al. 1936).

Another important characteristic that is the progressive burning rate (Department of the Army 1984; Meyer et al. 2007; Valença et al. 2013). This is possible because, while a powder grain burns from the outside towards the inside, it reaches different layers with higher burning rates, as well as higher temperatures. The modifying agents and surface coats burn first, and then the inner part of the grain, made of nitrocellulose (which has a higher burning heat), is reached. A progressive burning is advantageous because it makes possible to keep the pressure inside the barrel high for a longer time, and a higher pressure peak is avoided. In general, the behavior of the burning allows the formation of a plateau at a high pressure, which gives the same overall energy under the curve if there was a single higher pressure peak. This is advantageous because 
the gun barrel will not have to tolerate very big pressures (U. S. Army 1964).

In small arms ammunition, particularly where progressive burning powder is used as the propellant, it often happens that the propellant charge does not completely burn inside the barrel and that combustion thereof is prolonged even after the projectile or shot charge has left the barrel. This prolonged combustion is objectionable because the burning particles of powder are expelled from the barrel of the arm behind the projectile, and the energy which would otherwise be imparted to the projectile is lost. In accordance with Olsen's invention (Olsen and Tibbitts 1938), the spherical powder charge is also clean burning and is completely consumed by combustion by the time the projectile or shot charge leaves the barrel whereby the objectionable conditions, such as deposits in the barrel and the ejection of luminous particles of powder from the muzzle of the gun, are substantially reduced.

\section{PRODUCTION}

Spherical smokeless powder is generally made from nitrocellulose as the base material. The nitrocellulose may be derived from purified cellulose, such as from cotton linters or wood, which is then nitrated by being treated with nitric and sulfuric acids (Olsen 1942; Valença et al. 2013; Department of the Army 1984).

In the manufacture of nitrocellulose, it is necessary to subject the nitrated cellulose to extensive purification treatments in order to remove the residual acid and unstable esters from the nitrocellulose fibers. A large amount of water is required for these purification treatments, and a considerable consumption of heat is necessary (Olsen 1942). In order to convert nitrocellulose, which has been purified, into gelatinized or colloided smokeless powder grains, it is customary to replace the water contained in the nitrocellulose with alcohol by means of a displacement process (Olsen et al. 1936).

Nitrocellulose which has deteriorated or contained centers of high acid concentration, as is frequently present in aged cannon powder, may be purified and stabilized to an extent sufficient to for use as propellant. This may be rendered suitable by grinding the deteriorated nitrocellulose powder in water slurry and thereafter agitating the slurry in a sufficient quantity of a solvent, such as water, to wash out and disperse the centers of high acidity. It is also desirable to add to the slurry a sufficient quantity of a neutralizing agent, such as calcium carbonate or other mild alkali, so as to neutralize the acid which may be washed out of the nitrocellulose spheres/pieces (Olsen et al. 1939, 1940).
Regarding the production of the smokeless powder, Olsen and his co-workers developed a process to manufacture a smokeless powder in a manner so as to secure a superior product irrespective (within limits) of the base. The base may be nitrocellulose, and its kind and character, as well as composition, may be varied; it may be wholly or partially purified fibrous nitrocellulose; it may be dense colloided nitrocellulose in the form of existing powder, or of good, poor or indifferent stability (Silk 1945; Olsen et al. 1936). Also, during the course of operations, the product is purified and stabilized, and the incorporation of additional ingredients, such as stabilizers and modifying agents (burning regulators or accelerators), are added (Olsen et al. 1936).

In accordance with Olsen's invention (Olsen et al. 1936), a greatly simplified procedure can be used for accomplishing the desired purification of the nitrocellulose as well as the gelatinization thereof and the formation of grains. If the nitrocellulose is impure and in an unstable condition, it is treated with a suitable solvent to form a lacquer from which the impurities are then removed by agitation in a suitable non-solvent bath, such as water. By distilling off a sufficient amount of the solvent during continued agitation, the gelatinized nitrocellulose becomes converted to preliminary grains. If the solvent for the powder base is selected so that it will be substantially immiscible with the non-solvent bath, and conditions tending to produce an emulsion with the powder base in the solution as the internal phase are maintained, the preliminary grains may be resolved into globular form and may, if desired, be completely solidified and employed as a propellant powder in this shape, without further operations of grain formation (Olsen 1942).

To protect the resulting powder against autocatalytic decomposition, one of the usual stabilizers as, for example, the centralities or diphenylamine, may be added to the lacquer so that the desired amount becomes incorporated within the individual particles of lacquer (Olsen 1942). The stabilizer is not only uniformly distributed throughout the globules formed within the medium and the grains thereafter formed from globules, but any acidic or deteriorated elements present will be distributed throughout the vehicle or medium (Olsen et al. 1936). The modifying agents such as deterrents or accelerators may also be incorporated with a grain and distributed throughout the grain in a manner similar to that of incorporating the stabilizer.

In general, the desired formation of the grains is accomplished by dispersing globules of the base (such as nitrocellulose) in a medium. This is accomplished by subjecting the base and a 
solvent to agitation in a non-solvent medium (such as water) so as to produce globules that are individually consolidated in order to form grains which are spherical (Carlucci and Jacobson 2008; Olsen et al. 1936; U. S. Army 1964). A volatile solvent may also be used to produce globules, being before vaporized by heating (Olsen et al. 1936). Suitable solvents include ethyl acetate, isopropyl acetate, methyl isobutyl ketone, as well as other organic liquids which are solvents for nitrocellulose not completely miscible with water (Coffee 1964; Silk 1945), and are sufficiently volatile so that they may be vaporized without having to resort to temperatures which are unsafe or inconvenient. The treatment with the solvent is preferably carried out to an extent sufficient to convert all of the nitrocellulose present in a lacquer (Olsen 1942).

The desired amount of solvent may be added to the slurry of nitrocellulose dust in the form of a spray and, in this manner, small globules of the solvent may be readily distributed throughout the mixture. During agitation and heating, the surfaces of the particles of nitrocellulose dust become softened due to the gelatinizing action of the solvent and tend to coalesce, forming clusters comprising a number of dust particles. This action may be explained since all liquid or plastic two-phase systems tend to a condition of minimum surface energy which can be accomplished by coalescence (Olsen et al. 1936, 1940).

The production of globules or the production of the quasi-emulsion may be secured in various ways. In accordance with Olsen's invention (Olsen et al. 1936), the base is dissolved in a solvent and the colloidal solution or lacquer is agitated rather violently in a non-solvent vehicle in the presence of the protective colloid and a neutralizer to form the globules. The protective colloid performs the function of effecting a quasiemulsion between the base and solvent and the non-solvent medium, with the base and solvent as the internal phase. The base is agitated with a solvent distributed in the non-solvent vehicle so as to form an emulsion with the medium as the internal phase; upon the addition of the protective colloid the emulsion is broken and under agitation inverted with the medium as the external phase. The neutralizer is added to remove the highacidity centers. A suitable neutralizer is calcium carbonate in the form of purified prepared chalk (Olsen et al. 1936, 1939).

The globules, which are distributed throughout the medium, are then subjected to treatment to extract the solvent, through distillation of the solvent secured by heating the medium to near the vaporizing point of the solvent. The distillation is so carried out as to effect a gradual vaporization of the solvent from the several globules; the process is preferably carried out by effecting vaporization at a rate decreasing from the beginning to the end of the vaporization period and at a rate less than the rate of diffusion of the solvent from the interiors to the exteriors of the globules. Such a distillation secures a solid grain as distinguished from one which is hollow and porous (Olsen et al. 1936).

After formation of the globules in the medium or vehicle, and after distillation of the solvent from the several globules to form grains, the medium is permitted to cool to a temperature sufficiently low to permit any modifying agent or stabilizer dissolved in the normally non-solvent medium to separate out at the lower temperature. In the case of the modifying agent, such as deterrent, it will become deposited on the grains so as to provide a surface treatment in the form of a coating or impregnation (Olsen et al. 1936). Figure 1 represents a manufacture process of spherical grains of smokeless powders.

The finishing operations are similar to the ones that are usually carried out during the manufacture of a single-base smokeless powder. After cooling of the medium, and when the grains have become consolidated or hardened, they may be subjected to a screening operation, in which the oversizes can be sent back for reworking and the screened grains can be subjected to a wringing operation and thereafter to a drying operation. The grains may be surface treated prior to drying with a suitable modifying

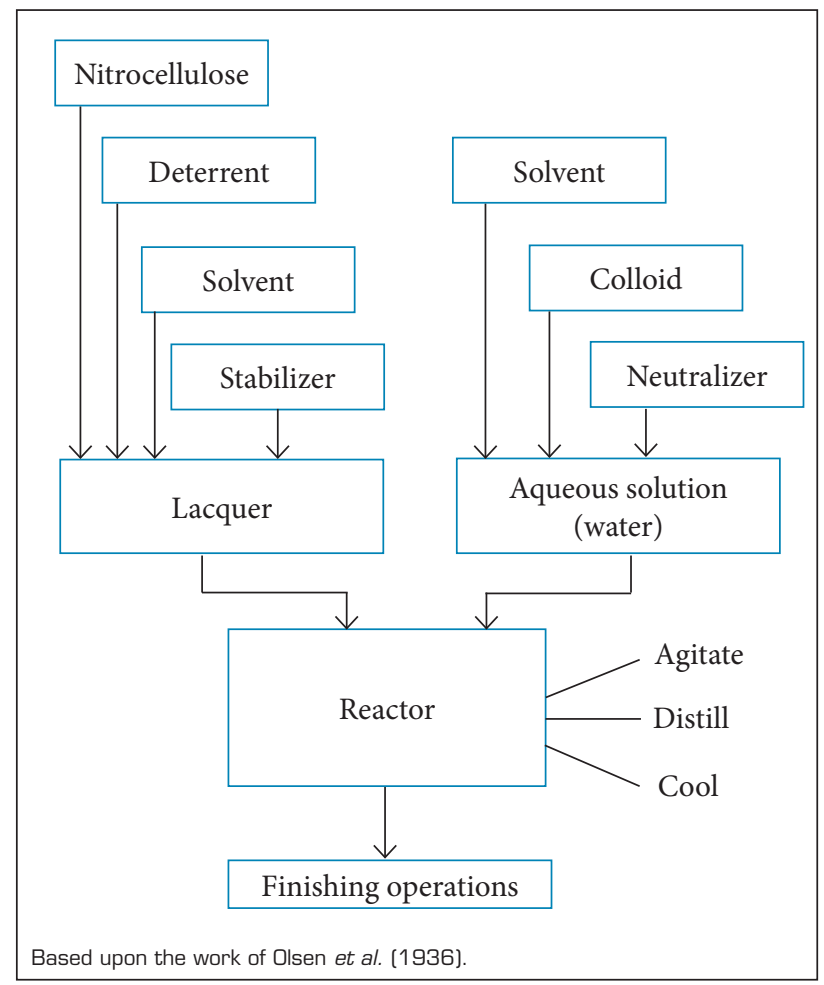

Figure 1. Spherical powder manufacturing process. 
agent, such as a deterrent or an accelerator. The coating may comprise nitroglycerin which acts as an accelerator; in that it allows the powder to be more readily ignitable and also acts as a waterproofing agent, rendering the powder non-hygroscopic. A suitable deterrent such as dibutylphthalate may be used along with the nitroglycerin or other accelerator and waterproofing agent. The grains may be then dried and glazed in the usual manner and thereafter blended if desired (Olsen et al. 1936, 1940).

The size of the grains is a function of the extent and the violence of the agitation as well as the amount of protective colloid and the viscosity of the dissolved base (Olsen et al. 1936; Valença et al. 2013). The grain size decreases with the increase of rotor speed, the increase of percentage of the colloid and the viscosity of the nitrocellulose. Furthermore the amount of solvent employed affects not only the size, shape and surface of the resultant grains, but also the gravimetric density thereof (Schaefer 1939; Olsen et al. 1940). Small amounts of solvent give a small rough cluster of particles as the grains while larger amounts of solvent give more spherical smoother grains. The rate at which the temperature is raised during evaporation of the solvent affects both the granulation and density of the resultant powder (Schaefer 1939).

The process for the manufacture of spherical powder, which Olsen and his co-workers have devised, combines nicely with Olsen's process for the quick stabilization of nitrocellulose to form a sequence of operations by which a finished powder may be produced more rapidly and more safely than by the usual process. It supplies a convenient means of making up a powder which contains nonvolatile solvents throughout the mass of the grains or deterrent or accelerant coatings upon their surface (Urbanski 1967).

Although the spherical powder starts as pure nitrocellulose, the finished product is a double-base propellant, since nitroglycerin is added after shaping and its content is created by surface impregnation.

\section{COMPARISON}

Table 1 provides a comparison between spherical powders and other common double-base smokeless powders with respect to manufacture process, characteristics and uses. It aims to briefly compare spherical powders against other double-base propellants. This is important since their production is more complex (hence expensive) than that of extruded propellants. Due to this, they are used mainly when the ammuntion does so requires.

Another important comparison aspect between propellants is the effectiveness and burning characteristics. This comparison is carried out using Fig. 2, which shows the difference between the thrust produced by propellants with different burning characteristics. Progressive burning occurs when the thrust produced increases with time and with distance traveled, inside the barrel (Kosanke 2012). During a regressive burning, the surface area decreases with time, and so does the thrust. The neutral burning happens when the burning area is approximately constant, which results in a constant thrust over the burning period. The pressure peak is higher and is reached first in a regressive burning rate because, as the burning area decreases, the gas production lowers, so the maximum pressure occurs in the very beginning of the barrel. On the other side, if a progressive burning propellant is used, the peak pressure is limited to a value that may be tolerated by the gun (Moss et al. 1983; U. S. Army 1964). It is also important to address the effect of adding high explosives in the composition on the propellant performance (Zhu et al. 2012).

Table 1. Comparison between spherical powders and common powders.

\begin{tabular}{|c|c|c|}
\hline Powder & Advantages & Disadvantages \\
\hline $\begin{array}{l}\text { Spherical } \\
\text { powder }\end{array}$ & $\begin{array}{l}\text { Unstable nitrocellulose can } \\
\text { be used. } \\
\text { Deteriorated nitrocellulose } \\
\text { from cannon powders can be } \\
\text { reused. } \\
\text { Loading spherical grains is } \\
\text { easier. } \\
\text { The grain formation is carried } \\
\text { in only one reactor. } \\
\text { Progressive burning rate. }\end{array}$ & $\begin{array}{l}\text { The plant to } \\
\text { produce cannot be } \\
\text { used to produce } \\
\text { other types of } \\
\text { powders. } \\
\text { Can only be used } \\
\text { in small caliber } \\
\text { ammunitions. }\end{array}$ \\
\hline $\begin{array}{l}\text { Other } \\
\text { double-base } \\
\text { powders }\end{array}$ & $\begin{array}{l}\text { Many physical forms can be } \\
\text { produced. } \\
\text { It can be used in bigger } \\
\text { ammunition calibers. } \\
\text { The same plant can be used to } \\
\text { produce different powders. }\end{array}$ & $\begin{array}{l}\text { High consumption } \\
\text { of water and } \\
\text { energy during the } \\
\text { production process. } \\
\text { Stable nitrocellulose } \\
\text { is necessary. }\end{array}$ \\
\hline
\end{tabular}

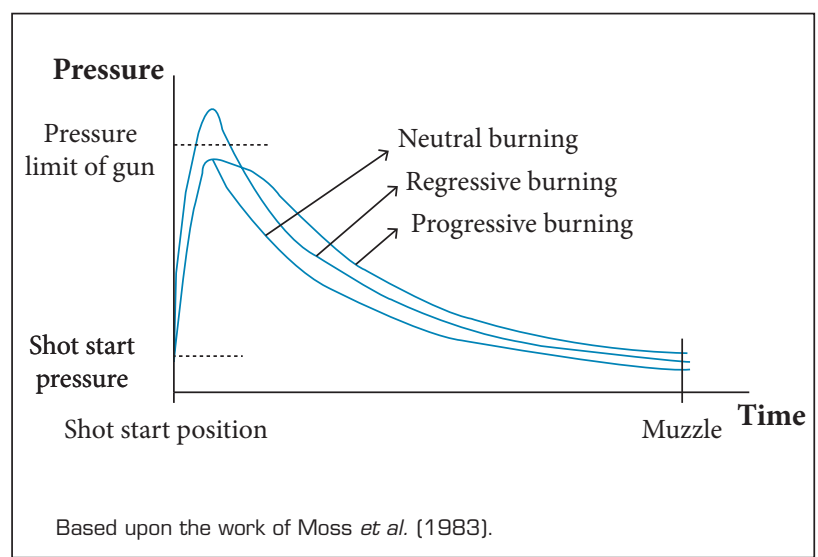

Figure 2. Pressure-time curves for different burning rates. 
In Fig. 2, "Shot start position" and "Muzzle" correspond to the time when these positions are reached. The curves shown in Fig. 2 can be determined using a closed vessel. The spherical powder can be used in small ammunitions to provide a greater muzzle velocity, which will result in a bigger kinetic energy being transferred to the target.

A typical peak pressure for a $5.56-\mathrm{mm}$ ammunition is $354 \mathrm{MPa}$ (Moss et al. 1983); it is considered when designing a new gun or barrel, hence this is the value considered to be compared against.

\section{CONCLUSION}

The great advantage of the spherical powder manufacture process is the possibility of using deteriorated and unstable nitrocellulose. Aged powders that would be destroyed can be reused, which results in a lower production cost. Also, the nitrocellulose stabilization process, that requires a big quantity of water and energy, is not necessary since the process of making the propellant spherical already stabilizes the nitrocellulose. Hence the process to make the powder spherical substitutes the stabilization process. One disadvantage is the fact that the spherical powder production plant cannot be used to manufacture other types of powder, due to the specificity of equipment and the lack of machinery to extrude propellants.

Regarding ballistic characteristics, the spherical powders are appropriate for use in small ammunitions (usually up to $50-\mathrm{mm}$ caliber rounds) because of their progressive burning rate and high loading density, which will result in a high muzzle speed.

It is therefore undeniable that they are very important in the propellants field and should not be neglected by ammunition and propellant industries. This is major when it comes to small rounds that benefit most from the burning behavior of the spherical powder.

\section{REFERENCES}

Akhavan J (2004) The chemistry of explosives. 2nd ed. Cambridge: RSC Paperbacks.

Barzanjy MJ [2011) Life cycle and performances progressing of artillery barrels. International Journal of Mechanical and Mechanics Engineering 11(6):19-22.

Boddicker FL, inventor; 1940 Aug 6. Process for the manufacture of smokeless powder. US $2210871 \mathrm{~A}$.

Carlucci DE, Jacobson SS (2008) Ballistics: theory and design of guns and ammunition. Boca Raton: CRC Press.

Coffee RE; inventor; 1964 Jan 21. Particulate nitrocellulose coated with sorbitan trioleate. US3118797 A.

Cooper P (1996) Explosives engineering. Weinheim: Wiley-VCH.

Department of the Army (1984). Military Explosives TM 9-1300214 - Department of the Army Technical Manual. Arlington County: Department of the Army Headquarters.

Heramb RM, McCord BR (2002) The manufacture of smokeless powders and their forensic analysis: a brief review; [accessed 2015 Nov 15]. https: / / www.ncjrs.gov/App/publications/abstract. aspx?|D=240495

Hodgdon Powder Co. (2014) A brief history of Winchester smokeless propellants; [accessed 2015 Nov 12]. http:/ / www.wwpowder.com/history.html

Kosanke K (2012) Terminology of model rocketry; [accessed 2015 Nov 15]. https://www.apogeerockets.com/education/downloads/Newsletter321.pdf

Meyer R, Kohler J, Homburg A (2007) Explosives. 6th ed. Weinheim: Wiley-VCH.

Moss GM, Farrar CL, Leeming DW (1983) Military ballistics: a basic manual. London: Pergamon Press.

Olsen F, inventor; 1942 Aug 11. Smokeless powder. US Patent 2292469.
Olsen F, Kerone EBW, Tibbitts GC, inventors; 1940 Jul 9. Manufacture of smokeless powders. US $2206916 \mathrm{~A}$.

Olsen F, Tibbitts GC, inventors; 1938 Mar 15. Propellant powder. US $2111075 \mathrm{~A}$.

Olsen F, Tibbitts GC, Kerone EBW, inventors; 1936 Jan 7. Manufacture of smokeless powders. US $2027114 \mathrm{~A}$.

Olsen F, Tibbitts GC, Kerone EBW, inventors; 1939 Oct 10. Manufacture of smokeless powder. US $2175212 \mathrm{~A}$.

Piper WE inventor; 1953 Oct 20. Manufacture of smokeless powder. US 2655694 A.

Schaefer HF, inventor; 1939 May 30. Explosive. US 2160626 A.

Silk CE, inventor; 1945 May 1. Smokeless powders process. US 2375175 A.

Tao CB, Zhang Y, Li S, Jia C, Li Y, Zhang X, He Z (2010) Mechanism of interior ballistic peak phenomenon of guns and its effects. J Appl Mech - T ASME 77(5):1-5. doi: 10.1115/1.4001561

U. S. Army (1964) AMCP 706-175 - Engineering design handbook. Washington: United States Army Materiel Command.

United Nations (2015) UN recommendations on the transport of dangerous goods - model regulations. 19th ed. New York: United Nations.

Urbanski T (1967) Chemistry and technology of explosives. vol. 3. Warszawa: Polish Scientific Publishers.

ValençaUS, ReisSS, PalazzoM, RochaJF, AthaydeAAC(2013)Engenharia dos explosivos: um enfoque dual. Rio de Janeiro: Fundação Ricardo Franco.

ZhuL, Ou JY, Yang XY, Wang YJ(2012)Effects composite spherical propellants on improvement of mechanical and combustion properties of modified double-based propellant. Hanneng Cailiao/Chinese Journal of Energetic Materials 20(5):592-595. doi: 10.3969/j.issn.1006-9941.2012.05.016 